\title{
SOME PRESERVATION PROPERTIES OF MKZ-STANCU TYPE OPERATORS
}

\author{
AYŞEGÜL ERENÇIN, GÜLEN BAŞCANBAZ-TUNCA AND FATMA TAŞDELEN
}

\begin{abstract}
In this work, we construct Stancu type modification of the generalization of Meyer-König and Zeller operators (MKZ) defined in [12]. We show that the Lipschitz constant of a Lipschitz continuous function and the properties of the function of modulus of continuity can be retained by these operators.
\end{abstract}

\section{INTRODUCTION}

In [13], by means of the probabilistic methods Stancu constructed a generalization of Bernstein polynomials as follows:

$$
S_{n}^{\alpha}(f ; x)=\sum_{k=0}^{n} f\left(\frac{k}{n}\right) s_{n, k}(x, \alpha), \quad x \in[0,1], n \in \mathbb{N},
$$

where $\alpha \geq 0$ is a parameter and

$$
s_{n, k}(x, \alpha):=\left(\begin{array}{l}
n \\
k
\end{array}\right) \frac{\prod_{i=0}^{k-1}(x+i \alpha) \prod_{j=0}^{n-k-1}(1-x+j \alpha)}{(1+\alpha)(1+2 \alpha) \cdots(1+(n-1) \alpha)} .
$$

Some works related to Stancu type generalization of some linear positive operators can be found in [2], [6], [7], [11], [14] and [19].

The Meyer-König and Zeller operators [10] are defined by

$$
M_{n}(f ; x)= \begin{cases}(1-x)^{n+1} \sum_{k=0}^{\infty} f\left(\frac{k}{n+k+1}\right)\left(\begin{array}{c}
n+k \\
k
\end{array}\right) x^{k} & , x \in[0,1) \\
f(x) & , x=1\end{cases}
$$

2010 Mathematics Subject Classification. 41A25, 41A36.

Key words and phrases. Function of modulus of continuity, Lipschitz continuous function, Stancu type operator . 
In order to get the monotonicity properties, Cheney and Sharma [4] introduced the following slight modification of these operators

$$
T_{n}(f ; x)=\left\{\begin{array}{ll}
(1-x)^{n+1} \sum_{k=0}^{\infty} f\left(\frac{k}{n+k}\right)\left(\begin{array}{c}
n+k \\
k
\end{array}\right) x^{k} & , x \in[0,1) \\
f(x) & , x=1
\end{array} .\right.
$$

In 2007, Rempulska and Skorupka [12] defined a generalization of MKZ operators on an unbounded interval as follows:

$$
M_{n}^{*}(f ; x)= \begin{cases}\left(1-\frac{x}{b_{n}}\right)^{n+1} \sum_{k=0}^{\infty} f\left(\frac{k}{n+k} b_{n}\right)\left(\begin{array}{c}
n+k \\
k
\end{array}\right)\left(\frac{x}{b_{n}}\right)^{k} & , x \in\left[0, b_{n}\right) \\
f(x) & , x \geq b_{n}\end{cases}
$$

where $n \in \mathbb{N}$ and $\left(b_{n}\right)$ is a sequence of real numbers having the properties

$$
1 \leq b_{n}<b_{n+1}, \lim _{n \rightarrow \infty} b_{n}=\infty, \lim _{n \rightarrow \infty} \frac{b_{n}}{n}=0
$$

The authors investigated some approximation properties of these operators for differentiable functions in polynomial weighted spaces. Very recently, Erençin, Ince and Olgun [5] proposed a modification of the operators given by (1.1) based on $q$-integers and obtained some convergence properties of these operators in weighted spaces of continuous functions on $[0, \infty)$ with the help of a weighted Korovkin type theorem. Furthermore, they also gave an application to functional differential equations and Stancu type remainder.

In this work, we introduce the following Stancu type generalization of the operators $M_{n}^{*}$ defined by (1.1)

$$
S_{n, \alpha}(f ; x)=\left\{\begin{array}{ll}
\sum_{k=0}^{\infty} f\left(\frac{k}{n+k} b_{n}\right) P_{n, k}(\alpha, x) & , x \in\left[0, b_{n}\right) \\
f(x) & , x \geq b_{n}
\end{array},\right.
$$

where $n \in \mathbb{N}, f$ is a continuous function on $[0, \infty),\left(b_{n}\right)$ is a sequence of real numbers satisfying the conditions given in (1.2), $\alpha \geq 0$ is a parameter and

$$
P_{n, k}(\alpha, x):=\left(\begin{array}{c}
n+k \\
k
\end{array}\right) \frac{\prod_{s=0}^{k-1}\left(\frac{x}{b_{n}}+\alpha s\right) \prod_{s=0}^{n}\left(1-\frac{x}{b_{n}}+\alpha s\right)}{\prod_{s=0}^{n+k}(1+\alpha s)} .
$$

An empty product in (1.4) will be taken as 1. It is obvious that for $\alpha=$ 0 the operators $S_{n, \alpha}$ reduce to the operators $M_{n}^{*}$. We remark that since $P_{n, 0}(\alpha, 0)=1$ and $P_{n, k}(\alpha, 0)=0$ for $k \geq 1$ we have $S_{n, \alpha}(f ; 0)=f(0)$. By taking into consideration this fact, a simple computation shows that the 
operators $S_{n, \alpha}$ for $\alpha>0$ can also be represented as

$$
S_{n, \alpha}(f ; x)= \begin{cases}f(0) & , x=0 \\ \int_{0}^{1} \psi_{\frac{1}{\alpha}, \frac{x}{b_{n}}}(t) M_{n}^{*}\left(f, t b_{n}\right) d t & , x \in\left(0, b_{n}\right), \\ f(x) & , x \geq b_{n}\end{cases}
$$

where the operators $M_{n}^{*}$ are given by (1.1) and

$$
\psi_{\frac{1}{\alpha}, \frac{x}{b_{n}}}(t)=\frac{t^{\frac{x}{\alpha b_{n}}-1}(1-t)^{\frac{b_{n}-x}{\alpha b_{n}}-1}}{B\left(\frac{x}{\alpha b_{n}}, \frac{b_{n}-x}{\alpha b_{n}}\right)}
$$

in which $B$ is the beta function defined by

$$
B(x, y)=\int_{0}^{1} t^{x-1}(1-t)^{y-1} d t=\frac{\Gamma(x) \Gamma(y)}{\Gamma(x+y)},(x, y>0) .
$$

Now let us consider the following operators

$$
B_{\alpha, b_{n}}(f ; x)=\int_{0}^{1} \psi_{\frac{1}{\alpha}, \frac{x}{b_{n}}}(t) f\left(t b_{n}\right) d t, \quad x \in\left(0, b_{n}\right),
$$

where $\psi_{\frac{1}{\alpha}, \frac{x}{b_{n}}}(t)$ is defined by (1.6). Straightforward computation yields

$$
B_{\alpha, b_{n}}(1 ; x)=1, B_{\alpha, b_{n}}(s ; x)=x .
$$

For future discussions, we recall the following definitions.

Let $f$ be a real valued continuous function defined on $[0, \infty)$. Then $f$ is said to be Lipschitz continuous of order $\mu$ on $[0, \infty)$ if

$$
|f(x)-f(y)| \leq A|x-y|^{\mu}
$$

for $x, y \in[0, \infty)$ with $A>0$ and $0<\mu \leq 1$. The set of Lipschitz continuous functions is denoted by $\operatorname{Lip}_{A}(\mu)$.

A real valued continuous and non-negative function $\omega$ defined on $[0, \infty)$ is called the function of modulus of continuity if each of the following conditions is satisfied:

a) $\omega\left(t_{1}+t_{2}\right) \leq \omega\left(t_{1}\right)+\omega\left(t_{2}\right)$ i.e., $\omega$ is semi-additive,

b) $\omega\left(t_{1}\right) \geq \omega\left(t_{2}\right)$ for $t_{1} \geq t_{2}$, i.e., $\omega$ is non-decreasing,

c) $\lim _{t \rightarrow 0^{+}} \omega(t)=\omega(0)=0$.

We now reproduce the following properties of the operators $M_{n}^{*}(f ; x):=$ $M_{n}^{*}(f)$ given by (1.1).

Lemma A ([18]). If $f \in \operatorname{Lip}_{A}(\mu)$, then for all $n \in \mathbb{N}, M_{n}^{*}(f) \in \operatorname{Lip}_{A}(\mu)$.

Lemma B ([18]). If $\omega$ is a function of modulus of continuity, then for all $n \in \mathbb{N}, M_{n}^{*}(\omega)$ is also a function of modulus of continuity. 


\section{MAIN RESUltS}

It is well known that first elementary proof regarding preservation of Lipschitz constant of a Lipschitz continuous function was given by Brown, Elliott and Paget in [1] for Bernstein polynomials. Similar results for other univariate or multivariate operators can be found in [3], [8],[15] [16] and [17]. On the other hand, in [9] Li established that Bernstein polynomials also preserve the properties of the general function of the modulus of continuity which is related to smoothness. Cao, Ding and $\mathrm{Xu}[3]$ extended for the first time this result to the multivariate Baskakov operators.

Our objective in this work is to show that the operators $S_{n, \alpha}(f ; x):=$ $S_{n, \alpha}(f)$ defined by (1.5) preserve the properties of the function of modulus of continuity and Lipschitz condition. For this purpose, let $\triangle$ be a set defined by

$$
\triangle:=\{(u, v): u \geq 0, v \geq 0, u+v \leq 1, u, v \in \mathbb{R}\} .
$$

For $\alpha>0$ and $x, y \in\left(0, b_{n}\right)$ such that $x<y$ we define the following auxiliary function

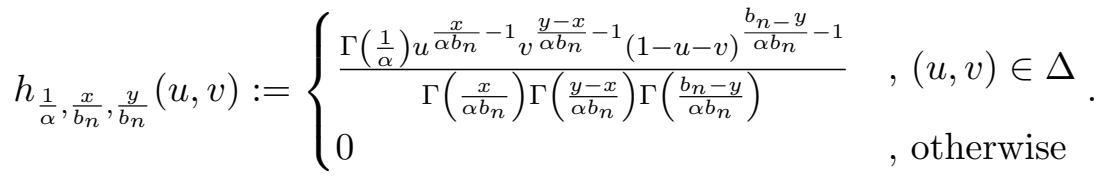

Theorem 1. For any $t \in(0,1)$ we have
(a) $\psi_{\frac{1}{\alpha}, \frac{x}{b_{n}}}(t)=\int_{0}^{1-t} h_{\frac{1}{\alpha}, \frac{x}{b_{n}}, \frac{y}{b_{n}}}(t, v) d v$
(b) $\psi_{\frac{1}{\alpha}, \frac{y}{b_{n}}}(t)=\int_{0}^{t} h_{\frac{1}{\alpha}, \frac{x}{b_{n}}, \frac{y}{b_{n}}}(u, t-u) d u$
(c) $\psi_{\frac{1}{\alpha}, \frac{y-x}{b_{n}}}(t)=\int_{0}^{1-t} h_{\frac{1}{\alpha}, \frac{x}{b_{n}}, \frac{y}{b_{n}}}(u, t) d u$,

where $\psi_{\frac{1}{\alpha}, \frac{x}{b_{n}}}$ is given by (1.6).

Proof. (a) From (2.1), one has

$$
\begin{aligned}
& \int_{0}^{1-t} h_{\frac{1}{\alpha}, \frac{x}{b_{n}}, \frac{y}{b_{n}}}(t, v) d v \\
= & \frac{\Gamma\left(\frac{1}{\alpha}\right) t^{\frac{x}{\alpha b_{n}}}-1}{\Gamma\left(\frac{x}{\alpha b_{n}}\right) \Gamma\left(\frac{y-x}{\alpha b_{n}}\right) \Gamma\left(\frac{b_{n}-y}{\alpha b_{n}}\right)} \int_{0}^{1-t} v^{\frac{y-x}{\alpha b_{n}}-1}(1-t-v)^{\frac{b_{n}-y}{\alpha b_{n}}-1} d v \\
= & \frac{\Gamma\left(\frac{1}{\alpha}\right) t^{\frac{x}{\alpha b_{n}}-1}(1-t)^{\frac{b_{n}-x}{\alpha b_{n}}-1}}{\Gamma\left(\frac{x}{\alpha b_{n}}\right) \Gamma\left(\frac{y-x}{\alpha b_{n}}\right) \Gamma\left(\frac{b_{n}-y}{\alpha b_{n}}\right)} \int_{0}^{1-t}\left(\frac{v}{1-t}\right)^{\frac{y-x}{\alpha b_{n}}-1}\left(1-\frac{v}{1-t}\right)^{\frac{b_{n}-y}{\alpha b_{n}}-1} \frac{d v}{1-t} .
\end{aligned}
$$


Now making the substitution $\xi=\frac{v}{1-t}$ and using the definition of beta function, we find

$$
\begin{aligned}
& \int_{0}^{1-t} h_{\frac{1}{\alpha}, \frac{x}{b_{n}}, \frac{y}{b_{n}}}(t, v) d v \\
= & \frac{\Gamma\left(\frac{1}{\alpha}\right) t^{\frac{x}{\alpha b_{n}}-1}(1-t)^{\frac{b_{n}-x}{\alpha b_{n}}-1}}{\Gamma\left(\frac{x}{\alpha b_{n}}\right) \Gamma\left(\frac{y-x}{\alpha b_{n}}\right) \Gamma\left(\frac{b_{n}-y}{\alpha b_{n}}\right)} \int_{0}^{1} \xi^{\frac{y-x}{\alpha b_{n}}-1}(1-\xi)^{\frac{b_{n}-y}{\alpha b_{n}}-1} d \xi \\
= & \frac{\Gamma\left(\frac{1}{\alpha}\right) t^{\frac{x}{\alpha b_{n}}-1}(1-t)^{\frac{b_{n}-x}{\alpha b_{n}}-1}}{\Gamma\left(\frac{x}{\alpha b_{n}}\right) \Gamma\left(\frac{y-x}{\alpha b_{n}}\right) \Gamma\left(\frac{b_{n}-y}{\alpha b_{n}}\right)} B\left(\frac{y-x}{\alpha b_{n}}, \frac{b_{n}-y}{\alpha b_{n}}\right) \\
= & \frac{\Gamma\left(\frac{1}{\alpha}\right) t^{\frac{x}{\alpha b_{n}}-1}(1-t)^{\frac{b_{n}-x}{\alpha b_{n}}-1}}{\Gamma\left(\frac{x}{\alpha b_{n}}\right) \Gamma\left(\frac{b_{n}-x}{\alpha b_{n}}\right)} \\
= & \frac{t^{\frac{x}{\alpha b_{n}}-1}(1-t)^{\frac{b_{n}-x}{\alpha b_{n}}-1}}{} \\
= & \psi_{\frac{1}{\alpha}, \frac{x}{b_{n}}}(t) .
\end{aligned}
$$

(b) Similarly,

$$
\begin{aligned}
& \int_{0}^{t} h_{\frac{1}{\alpha}, \frac{x}{b_{n}}, \frac{y}{b_{n}}}(u, t-u) d u \\
= & \frac{\Gamma\left(\frac{1}{\alpha}\right)(1-t)^{\frac{b_{n}-y}{\alpha b_{n}}-1}}{\Gamma\left(\frac{x}{\alpha b_{n}}\right) \Gamma\left(\frac{y-x}{\alpha b_{n}}\right) \Gamma\left(\frac{b_{n}-y}{\alpha b_{n}}\right)} \int_{0}^{t} u^{\frac{x}{\alpha b_{n}}-1}(t-u)^{\frac{y-x}{\alpha b_{n}}-1} d u \\
= & \frac{\Gamma\left(\frac{1}{\alpha}\right) t^{\frac{y}{\alpha b_{n}}-1}(1-t)^{\frac{b_{n}-y}{\alpha b_{n}}-1}}{\Gamma\left(\frac{x}{\alpha b_{n}}\right) \Gamma\left(\frac{y-x}{\alpha b_{n}}\right) \Gamma\left(\frac{b_{n}-y}{\alpha b_{n}}\right)} \int_{0}^{t}\left(\frac{u}{t}\right)^{\frac{x}{\alpha b_{n}}-1}\left(1-\frac{u}{t}\right)^{\frac{y-x}{\alpha b_{n}}-1} \frac{d u}{t} .
\end{aligned}
$$

If we make the substitution $\xi=\frac{u}{t}$, then we get

$$
\begin{aligned}
& \int_{0}^{t} h_{\frac{1}{\alpha}, \frac{x}{b_{n}}, \frac{y}{b_{n}}}(u, t-u) d u \\
= & \frac{\Gamma\left(\frac{1}{\alpha}\right) t^{\frac{y}{\alpha b_{n}}-1}(1-t)^{\frac{b_{n}-y}{\alpha b_{n}}-1}}{\Gamma\left(\frac{x}{\alpha b_{n}}\right) \Gamma\left(\frac{y-x}{\alpha b_{n}}\right) \Gamma\left(\frac{b_{n}-y}{\alpha b_{n}}\right)} \int_{0}^{1} \xi^{\frac{x}{\alpha b_{n}}-1}(1-\xi)^{\frac{y-x}{\alpha b_{n}}-1} d \xi \\
= & \frac{\Gamma\left(\frac{1}{\alpha}\right) t^{\frac{y}{\alpha b_{n}}-1}(1-t)^{\frac{b_{n}-y}{\alpha b_{n}}-1}}{\Gamma\left(\frac{x}{\alpha b_{n}}\right) \Gamma\left(\frac{y-x}{\alpha b_{n}}\right) \Gamma\left(\frac{b_{n}-y}{\alpha b_{n}}\right)} B\left(\frac{x}{\alpha b_{n}}, \frac{y-x}{\alpha b_{n}}\right)
\end{aligned}
$$




$$
\begin{aligned}
& =\frac{\Gamma\left(\frac{1}{\alpha}\right) t^{\frac{y}{\alpha b_{n}}-1}(1-t)^{\frac{b_{n}-y}{\alpha b_{n}}-1}}{\Gamma\left(\frac{y}{\alpha b_{n}}\right) \Gamma\left(\frac{b_{n}-y}{\alpha b_{n}}\right)} \\
& =\psi_{\frac{1}{\alpha}, \frac{y}{b_{n}}}(t),
\end{aligned}
$$

which is the required result.

(c) By means of the equality (2.1) we can write

$$
\begin{aligned}
& \int_{0}^{1-t} h_{\frac{1}{\alpha}, \frac{x}{b_{n}}, \frac{y}{b_{n}}}(u, t) d u \\
= & \frac{\Gamma\left(\frac{1}{\alpha}\right) t^{\frac{y-x}{\alpha b_{n}}-1}}{\Gamma\left(\frac{x}{\alpha b_{n}}\right) \Gamma\left(\frac{y-x}{\alpha b_{n}}\right) \Gamma\left(\frac{b_{n}-y}{\alpha b_{n}}\right)} \int_{0}^{1-t} u^{\frac{x}{\alpha b_{n}}-1}(1-t-u)^{\frac{b_{n}-y}{\alpha b_{n}}-1} d u \\
= & \frac{\Gamma\left(\frac{1}{\alpha}\right) t^{\frac{y-x}{\alpha b_{n}}-1}(1-t)^{\frac{b_{n}-(y-x)}{\alpha b_{n}}}-1}{\Gamma\left(\frac{x}{\alpha b_{n}}\right) \Gamma\left(\frac{y-x}{\alpha b_{n}}\right) \Gamma\left(\frac{b_{n}-y}{\alpha b_{n}}\right)} \int_{0}^{1-t}\left(\frac{u}{1-t}\right)^{\frac{x}{\alpha b_{n}}-1}\left(1-\frac{u}{1-t}\right)^{\frac{b_{n}-y}{\alpha b_{n}}-1} \frac{d u}{1-t} .
\end{aligned}
$$

Thus letting $\xi=\frac{u}{1-t}$ we find

$$
\begin{aligned}
& \int_{0}^{1-t} h_{\frac{1}{\alpha}, \frac{x}{b_{n}}, \frac{y}{b_{n}}}(u, t) d u \\
= & \frac{\Gamma\left(\frac{1}{\alpha}\right) t^{\frac{y-x}{\alpha b_{n}}-1}(1-t)^{\frac{b_{n}-(y-x)}{\alpha b_{n}}-1}}{\Gamma\left(\frac{x}{\alpha b_{n}}\right) \Gamma\left(\frac{y-x}{\alpha b_{n}}\right) \Gamma\left(\frac{b_{n}-y}{\alpha b_{n}}\right)} \int_{0}^{1} \xi^{\frac{x}{\alpha b_{n}}-1}(1-\xi)^{\frac{b_{n}-y}{\alpha b_{n}}-1} d \xi \\
= & \frac{\Gamma\left(\frac{1}{\alpha}\right) t^{\frac{y-x}{\alpha b_{n}}-1}(1-t)^{\frac{b_{n}-(y-x)}{\alpha b_{n}}-1}}{\Gamma\left(\frac{x}{\alpha b_{n}}\right) \Gamma\left(\frac{y-x}{\alpha b_{n}}\right) \Gamma\left(\frac{x}{\alpha b_{n}}\right)} B\left(\frac{b_{n}-y}{\alpha b_{n}}, \frac{b_{n}-y}{\alpha b_{n}}\right) \\
= & \frac{\Gamma\left(\frac{1}{\alpha}\right) t^{\frac{y-x}{\alpha b_{n}}-1}(1-t)^{\frac{b_{n}-(y-x)}{\alpha b_{n}}-1}}{\Gamma\left(\frac{y-x}{\alpha b_{n}}\right) \Gamma\left(\frac{b_{n}-(y-x)}{\alpha b_{n}}\right)} \\
= & \psi_{\frac{1}{\alpha}, \frac{y-x}{b_{n}}}(t)
\end{aligned}
$$

which completes the proof.

Theorem 2. If $f \in \operatorname{Lip}_{A}(\mu)$, then $S_{n, \alpha}(f) \in \operatorname{Lip}_{A}(\mu)$ for all $n \in \mathbb{N}$ and $\alpha>0$.

Proof. Let $x, y \in\left(0, b_{n}\right)$ such that $x<y$. Using items $(b)$ and $(a)$ of Theorem 1 , and taking into consideration the representation of the operators $S_{n, \alpha}$ 
given by (1.5) one gets

$$
\begin{aligned}
S_{n, \alpha}(f ; y)-S_{n, \alpha}(f ; x)= & \int_{0}^{1}\left[\psi_{\frac{1}{\alpha}, \frac{y}{b_{n}}}(t)-\psi_{\frac{1}{\alpha}, \frac{x}{b_{n}}}(t)\right] M_{n}^{*}\left(f ; t b_{n}\right) d t \\
= & \int_{0}^{1} \int_{0}^{t} h_{\frac{1}{\alpha}, \frac{x}{b_{n}}, \frac{y}{b_{n}}}(u, t-u) M_{n}^{*}\left(f ; t b_{n}\right) d u d t \\
& -\int_{0}^{1} \int_{0}^{1-t} h_{\frac{1}{\alpha}, \frac{x}{b_{n}}, \frac{y}{b_{n}}}(t, v) M_{n}^{*}\left(f ; t b_{n}\right) d v d t \\
= & \int_{0}^{1} \int_{u}^{1} h_{\frac{1}{\alpha}, \frac{x}{b_{n}}, \frac{y}{b_{n}}}(u, t-u) M_{n}^{*}\left(f ; t b_{n}\right) d t d u \\
& -\int_{0}^{1} \int_{0}^{1-t} h_{\frac{1}{\alpha}, \frac{x}{b_{n}}, \frac{y}{b_{n}}}(t, v) M_{n}^{*}\left(f ; t b_{n}\right) d v d t .
\end{aligned}
$$

Now letting $t-u=v$ and $t=u$ in the first and second integral, respectively, on the right side of the above equality we obtain

$$
\begin{aligned}
& S_{n, \alpha}(f ; y)-S_{n, \alpha}(f ; x) \\
= & \int_{0}^{1} \int_{0}^{1-u} h_{\frac{1}{\alpha}, \frac{x}{b_{n}}, \frac{y}{b_{n}}}(u, v) M_{n}^{*}\left(f ;(u+v) b_{n}\right) d v d u \\
& -\int_{0}^{1} \int_{0}^{1-u} h_{\frac{1}{\alpha}, \frac{x}{b_{n}}, \frac{y}{b_{n}}}(u, v) M_{n}^{*}\left(f ; u b_{n}\right) d v d u \\
= & \int_{0}^{1} \int_{0}^{1-u} h_{\frac{1}{\alpha}, \frac{x}{b_{n}}, \frac{y}{b_{n}}}(u, v)\left[M_{n}^{*}\left(f ;(u+v) b_{n}\right)-M_{n}^{*}\left(f ; u b_{n}\right)\right] d v d u
\end{aligned}
$$

so that

$$
\begin{aligned}
& \left|S_{n, \alpha}(f ; y)-S_{n, \alpha}(f ; x)\right| \\
\leq & \int_{0}^{1} \int_{0}^{1-u} h_{\frac{1}{\alpha}, \frac{x}{b_{n}}, \frac{y}{b_{n}}}(u, v)\left|M_{n}^{*}\left(f ;(u+v) b_{n}\right)-M_{n}^{*}\left(f ; u b_{n}\right)\right| d v d u .
\end{aligned}
$$

From Lemma A we have $M_{n}^{*}(f) \in \operatorname{Lip}_{A}(\mu)$. So using this fact and the item $(c)$ of Theorem 1 it follows that

$$
\begin{aligned}
\left|S_{n, \alpha}(f ; y)-S_{n, \alpha}(f ; x)\right| & \leq A \int_{0}^{1} \int_{0}^{1-u}\left(v b_{n}\right)^{\mu} h_{\frac{1}{\alpha}, \frac{x}{b_{n}}, \frac{y}{b_{n}}}(u, v) d v d u \\
& =A \int_{0}^{1} \int_{0}^{1-v}\left(v b_{n}\right)^{\mu} h_{\frac{1}{\alpha}, \frac{x}{b_{n}}, \frac{y}{b_{n}}}(u, v) d u d v \\
& =A \int_{0}^{1}\left(v b_{n}\right)^{\mu}\left[\int_{0}^{1-v} h_{\frac{1}{\alpha}, \frac{x}{b_{n}}, \frac{y}{b_{n}}}(u, v) d u\right] d v \\
& =A \int_{0}^{1}\left(v b_{n}\right)^{\mu} \psi_{\frac{1}{\alpha}, \frac{y-x}{b_{n}}}(v) d v .
\end{aligned}
$$


Finally by Hölder's inequality and the equations (1.7) and (1.8) we obtain that

$$
\begin{aligned}
\left|S_{n, \alpha}(f ; y)-S_{n, \alpha}(f ; x)\right| & \leq A\left(\int_{0}^{1} \psi_{\frac{1}{\alpha}, \frac{y-x}{b_{n}}}(v)\left(v b_{n}\right) d v\right)^{\mu} \\
& =A\left(B_{\alpha, b_{n}}(s ; y-x)\right)^{\mu} \\
& =A(y-x)^{\mu} .
\end{aligned}
$$

This is the desired result. Similarly, by using (1.5) it can be shown that for the cases $x=0, y \in\left(0, b_{n}\right) ; x=0, y \geq b_{n}$ and $x \in\left(0, b_{n}\right), y \geq b_{n}$ our claim is true. Thus the proof is completed.

Theorem 3. Let $\omega$ be a modulus of continuity function, then the operators $S_{n, \alpha}(\omega)$ have the same property for all $n \in \mathbb{N}$ and $\alpha>0$.

Proof. By (2.2), we have

$$
\begin{aligned}
& S_{n, \alpha}(\omega ; y)-S_{n, \alpha}(\omega ; x) \\
& =\int_{0}^{1} \int_{0}^{1-u} h_{\frac{1}{\alpha}, \frac{x}{b_{n}}, \frac{y}{b_{n}}}(u, v)\left[M_{n}^{*}\left(\omega ;(u+v) b_{n}\right)-M_{n}^{*}\left(\omega ; u b_{n}\right)\right] d v d u .
\end{aligned}
$$

From Lemma B the above equality leads to

$$
S_{n, \alpha}(\omega ; y)-S_{n, \alpha}(\omega ; x) \geq 0
$$

for $y>x$. Again from Lemma B we know that $M_{n}^{*}(\omega)$ is semi-additive. So, we may write in view of Theorem $1,(c)$ and (1.5) that

$$
\begin{aligned}
& S_{n, \alpha}(\omega ; y)-S_{n, \alpha}(\omega ; x) \\
& =\int_{0}^{1} \int_{0}^{1-u} h_{\frac{1}{\alpha}, \frac{x}{b_{n}}, \frac{y}{b_{n}}}(u, v)\left[M_{n}^{*}\left(\omega ;(u+v) b_{n}\right)-M_{n}^{*}\left(\omega ; u b_{n}\right)\right] d v d u \\
& \leq \int_{0}^{1} \int_{0}^{1-u} h_{\frac{1}{\alpha}, \frac{x}{b_{n}}, \frac{y}{b_{n}}}(u, v) M_{n}^{*}\left(\omega ; v b_{n}\right) d v d u \\
& =\int_{0}^{1} \int_{0}^{1-v} h_{\frac{1}{\alpha}, \frac{x}{b_{n}}, \frac{y}{b_{n}}}(u, v) M_{n}^{*}\left(\omega ; v b_{n}\right) d u d v \\
& =\int_{0}^{1} M_{n}^{*}\left(\omega ; v b_{n}\right)\left[\int_{0}^{1-v} h_{\frac{1}{\alpha}, \frac{x}{b_{n}}, \frac{y}{b_{n}}}(u, v) d u\right] d v \\
& =\int_{0}^{1} \psi_{\frac{1}{\alpha}, \frac{y-x}{b_{n}}}(v) M_{n}^{*}\left(\omega ; v b_{n}\right) d v \\
& =S_{n}(\omega ; y-x)
\end{aligned}
$$

which shows the semi-additivity of $S_{n, \alpha}(\omega ; x)$. Moreover, by the definition of $S_{n, \alpha}$ we have $\lim _{x \longrightarrow 0^{+}} S_{n, \alpha}(\omega ; x)=\omega(0)=0$. This completes the proof. 


\section{REFERENCES}

[1] B. M. Brown, D. Elliott and D. F. Paget, Lipschitz constants for the Bernstein polynomials of a Lipschitz continuous function, J. Approx. Theory, 49 (2) (1987), 196-199.

[2] İ. Büyükyazici, Approximation by Stancu-Chlodowsky polynomials, Comput. Math. Appl., 59 (1) (2010), 274-282.

[3] Feilong Cao, Chunmei Ding and Zongben Xu, On multivariate Baskakov operators, J. Math. Anal. Appl., 307 (1) (2005), 274-291.

[4] E. W. Cheney and A. Sharma, Bernstein power series, Canad. J. Math., 16 (1964), $241-253$.

[5] A. Erençin, H. G. Ince and A. Olgun, A class of linear positive operators in weighted spaces, Math. Slovaca, 62 (1) (2012), 63-76.

[6] Z. Finta, Direct and converse results for Stancu operator, Period. Math. Hungar., 44 (1) (2002), 1-6.

[7] S. G. Gal, Approximation by complex Berstein-Kantorovich and Stancu-Kantorovich polynomials and their iterates in compact disk, Rev. Anal. Numér. Théor. Approx., 37 (2) (2008), 159-168.

[8] M. K. Khan, Approximation properties of Beta operators, Progress in Approximation Theory, (1991), Academic Press, Boston, MA, (1991), 483-495.

[9] Zhongkai Li, Bernstein polynomials and modulus of continuity, J. Approx. Theory, 102 (2000), 171-174.

[10] W. Meyer-König and K. Zeller, Bernsteinsche potenzreihen, Studia Math., 19 (1960), 89-94.

[11] G. Nowak, Approximation properties for generalized q-Bernstein polynomials, J. Math. Anal. Appl., 350 (1) (2009), 50-55.

[12] L. Rempulska and M. Skorupka, Approximation by generalized MKZ-operators in polynomial weighted spaces, Anal. Theory Appl., 23 (1) (2007), 64-75.

[13] D. D. Stancu, Approximation of functions by a new class of linear polynomial operators, Rev. Roumaine Math. Pures Appl., 13 (1968), 1173-1194.

[14] E. I. Stoica-Laze, On the Stancu type linear positive operators of approximation constructed by using the beta and gamma functions, Stud. Univ. Babeş-Bolyai Math., $54(2)(2009), 117-126$.

[15] T. Trif, An Elementary proof of the preservation of Lipschitz constants by the Meyer-König and Zeller operators, J. Inequal. Pure Appl. Math., 4 (5) (2003), Article 90, 3pp.

[16] G. Başcanbaz-Tunca and Y. Tuncer, Some properties of multivariate beta operator, Fasc. Math., 41 (2009), 31-43.

[17] G. Başcanbaz-Tunca and Y. Tuncer, On a Chlodovsky variant of a multivariate beta operator, J. Comput. Appl. Math., 235 (16) (2011), 4816-4824.

[18] G. Başcanbaz-Tunca and F. Taşdelen, On Chlodovsky form of the Meyer-König and Zeller operators, An. Univ. Vest Timiş. Ser. Mat.-Inform., XLIX, 2 (2011), $137-144$.

[19] Jiang Yanjie, Li Junming, The rate of convergence of q-Bernstein-Stancu polynomials, Int. J. Wavelets, Multiresol. Info. Proc., 7 (6) (2009), 773-779.

(Received: February 20, 2013)

(Revised: April 17, 2013)
Ayşegül Erençin

Department of Mathematics

Abant İzet Baysal University 
Faculty of Arts and Sciences 14280, Bolu

Turkey

erencina@hotmail.com

Gülen Başcanbaz-Tunca and Fatma Taşdelen

Department of Mathematics

Faculty of Science

Ankara University

06100, Tandoan, Ankara

Turkey

tunca@science.ankara.edu.tr tasdelen@science.ankara.edu.tr 